\title{
Instrumento para evaluar los conocimientos matemáticos previos para la enseñanza del concepto de límite durante la pandemia SARS-CoV-2
}

\author{
Instrument for evaluating prior mathematical knowledge for the teaching \\ of Limit concept during the SARS-CoV-2 pandemic
}

\section{Instrumento para avaliar o conhecimento matemático prévio para o ensino do conceito de limite durante a pandemia de SARS-CoV-2}

Erick Radaí Rojas Maldonado

Universidad Michoacana de San Nicolás de Hidalgo, México errojas@umich.mx https://orcid.org/0000-0003-2521-5107

Jaqueline Toscano Galeana

Universidad Michoacana de San Nicolás de Hidalgo, México jaqueline.toscano@umich.mx https://orcid.org/0000-0001-9333-8956

\section{Resumen}

El objetivo del presente trabajo fue diseñar un instrumento que permitiera determinar, en el marco de la actual emergencia sanitaria generada por el virus SARS-CoV-2, los conocimientos matemáticos previos en torno al concepto límite de alumnos del bachillerato de Ingeniería y Arquitectura (sección 1) del Colegio Primitivo y Nacional de San Nicolás de Hidalgo. Para el diseño del instrumento se siguieron las cuatro fases de construcción que establece Soriano Rodríguez (2015), es decir, 1) objetivos, teoría y constructo, 2) validación juicio de expertos, 3) prueba piloto y 4) validación psicométrica. Los resultados de esa última fase demuestran que el instrumento se puede reproducir en diferentes muestras sin que constituyan un gran sesgo por parte de los participantes. Además, los puntajes de cada 


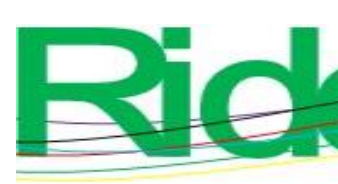

Revista Iberoamericana para la Investigación y el Desarrollo Educativo ISSN $2007-7467$

pregunta fueron consistentes con el puntaje total del instrumento. En conclusión, se puede afirmar que esta puede ser una herramienta muy útil no solo para detectar los conocimientos previos que los alumnos tienen en cuanto al cálculo diferencial, sino principalmente para crear estrategias didácticas que permitan atender esas debilidades a partir de los estilos de aprendizaje de los estudiantes.

Palabras clave: cálculo diferencial, enseñanza emergente, exploración, diagnóstico, límite.

\section{Abstract}

The technology that we have today is not conceivable without one of the pillar concepts in the construction of the Differential Calculus, the Limit. An important concept that, since its “discovery or invention" by Newton and Leibniz, has created difficulties for many mathematicians to understand it, it was not until the arrival of Cauchy that he defined it in a more practical way, but not for students, since various studies refer to the difficulty it has the study of Limit as well as the misconceptions that the student gives it and the little success on the part of the students in their understanding in which didactic strategies have been carried out for their understanding, all in a face-to-face setting. However, for reasons of SARS-CoV2 , the situation is even more difficult since everything is framed in an emerging teaching. Therefore, it is decided to design and create a diagnostic instrument of the student's previous knowledge that precedes the learning of Differential Calculus Limit in such a way that it measures, characterizes and synthetically explores both the procedural and conceptual skills of the cognitive processes of arithmetic and algebra, such that it allows to have enough constructs to understand the concept of limit. The instrument is validated in four phases in which the judgment of experts and the psychometric processes that allow the generation of scientific evidence are contemplated. This instrument conjectures the weaknesses that students may have and provides the teacher with a horizon to decide the didactic methodology to address the topic. With this result, it joins the efforts of various researchers in educational mathematics who seek to improve both the teaching and learning of the concept of limit.

Keywords: differential calculus, emerging learning, previous knowledge, diagnosis, limit. 


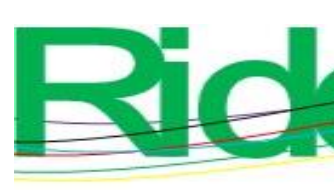

Revista Iberoamericana para la Investigación y el Desarrollo Educativo ISSN $2007-7467$

\section{Resumo}

O objetivo deste trabalho foi delinear um instrumento que permitisse determinar, no quadro da atual emergência sanitária gerada pelo vírus SARS-CoV-2, o conhecimento matemático prévio em torno do conceito de limite de alunos do Bacharelado em Engenharia e. Arquitetura (seção 1) da Escola Primitiva e Nacional de San Nicolás de Hidalgo. Para a concepção do instrumento, foram seguidas as quatro fases de construção estabelecidas por Soriano Rodríguez (2015), ou seja, 1) objetivos, teoria e construto, 2) validação do julgamento pericial, 3) teste piloto e 4) validação psicométrica. Os resultados desta última fase mostram que o instrumento pode ser reproduzido em diferentes amostras sem constituir um grande viés por parte dos participantes. Além disso, as pontuações de cada questão foram consistentes com a pontuação total do instrumento. En conclusión, se puede afirmar que esta puede ser una herramienta muy útil no solo para detectar los conocimientos previos que los alumnos tienen en cuanto al cálculo diferencial, sino principalmente para crear estrategias didácticas que permitan atender esas debilidades a partir de los estilos de aprendizaje de os estudantes.

Palavras-chave: cálculo diferencial, ensino emergente, exploração, diagnóstico, limite. Fecha Recepción: Agosto 2020 Fecha Aceptación: Mayo 2021

\section{Introducción}

Debido a los problemas ocasionados por la covid-19, actualmente nos encontramos en una etapa de reconstrucción de la enseñanza y del aprendizaje, la cual ha tenido que sustentarse en estrategias de enseñanza remota de emergencia para atender las necesidades educativas de la población. Como lo cometan Hodges, Moore, Lockee, Trust y Bond (2020), el objetivo principal ha sido proporcionar acceso a la instrucción, así como apoyos pedagógicos que permitan dar continuidad a las actividades educativas y capacitación a los profesores para impulsar el trabajo colaborativo y la enseñanza en entornos virtuales.

Sin embargo, según datos de la Organización de las Naciones Unidas para la Educación, la Ciencia y la Cultura (Unesco, 2020), la actual emergencia sanitaria ha provocado que casi $85 \%$ de los alumnos a nivel mundial no pueda desarrollar normalmente sus actividades académicas debido, principalmente, a problemas relacionados el escaso 


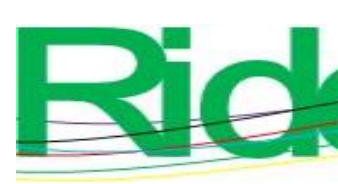

Revista Iberoamericana para la
Investigación y el Desarrollo Educativo
ISSN $2007-7467$

acceso a las tecnologías de la comunicación. En México, por ejemplo, según la Encuesta Nacional sobre la Disponibilidad y Uso de Tecnologías de la Información en los Hogares (Endutih) (Instituto Nacional de Estadística Geografía e Informática [Inegi], 2018), solo 47 $\%$ de los habitantes tiene conexión a internet, 52.4 millones de personas viven en situación de pobreza y 9.3 millones en pobreza extrema. Además, 21.1 millones de personas sufren rezago educativo (Consejo Nacional de Evaluación [Coneval], 2018).

Esta realidad es preocupante porque suele afectar con más crudeza a las poblaciones vulnerables (Rojas Maldonado, 2020a), las cuales suelen quedar a la expectativa de las iniciativas humanitarias llevadas a cabo por el Estado. En el caso de México, las estrategias políticas del gobierno en materia educativa para encarar la emergencia sanitaria se han enfocado en procurar que las actividades escolares se mantengan de forma virtual. Sin embargo, la cotidianidad ha demostrado que tanto para profesores y como para estudiantes el proceso de enseñanza y aprendizaje se ha visto entorpecido de manera considerable.

Por este motivo, el objetivo del presente trabajo fue diseñar un instrumento que permitiera determinar, en el marco de la actual emergencia sanitaria generada por el virus SARS-CoV-2, los conocimientos matemáticos previos en torno al concepto límite de alumnos del bachillerato de Ingeniería y Arquitectura (sección 1) del Colegio Primitivo y Nacional de San Nicolás de Hidalgo.

El concepto de límite resulta esencial para la construcción del cálculo diferencial, para encarar con éxito los estudios universitarios relacionados con la matemática y para impulsar la comprensión y el desarrollo tecnológico en la que se basa actualmente nuestra civilización (Cottrill et al., 1996), y ha sido abordado por algunos autores (Artigue, 1998; Cornu, 1991), quienes han demostrado las incorrectas apreciaciones de los alumnos sobre dicho concepto. Para mejorar su comprensión se han aplicado diversas estrategias que se han apoyado en herramientas tecnológicas, las cuales han permitido conseguir resultados ligeramente superiores a los alcanzados con la enseñanza tradicional (Rojas Maldonado, 2015, 2016, 2019). 


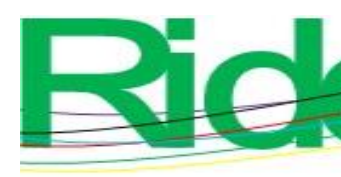

Revista Iberoan
Investigación $y$ el
ISSN 20

De forma general, se puede afirmar que si bien existen investigaciones que se pudieran vincular con el objetivo principal del presente trabajo, en realidad son pocas las que se enfocan en el estudio de los conocimientos previos de los estudiantes en torno al concepto límite en medio de una situación tan particular como la generada por la actual pandemia. Aun así, se pueden rescatar indagaciones como las de Mota Villegas y Valles Pereira (2015), quienes no solo explican la importancia del estudio estructurado de los conocimientos matemáticos previos de alumnos que comienzan en la universidad, sino que también demuestran lo incompleto que pueden ser los instrumentos usados hoy en día para medir tales conocimientos.

Por su parte, Urbina (2009) afirma que la evaluación de los conocimientos iniciales ayuda al docente a determinar el grado de profundidad con que se debe tratar un tema, así como las estrategias que puede emplear para que el alumno asimile los nuevos significados del objeto de aprendizaje ofrecido. $\mathrm{Al}$ respecto, el referido autor afirma lo siguiente:

Durante los últimos 20 años, las investigaciones en educación matemática han estado marcadas por el paradigma constructivista. Las ideas claves de este paradigma provienen o tienen sus raíces en investigaciones de muchos autores, entre los cuales se destacan: Piaget, Wallon, Vygotsky, Bruner, Dewey, Gagné, Ausubel, Novak y Henesian, entre otros. Todos ellos han coincidido en que aprender cualquier contenido escolar supone desde la concepción constructivista, atribuir un sentido y construir los significados implicados en dicho contenido, y que esta construcción no se lleva a cabo partiendo de cero (Urbina, 2009, p. 2).

En este sentido, Pino-Fan, Godino y Vincenç (2013) diseñan y aplican un instrumento para explorar la faceta epistémica del conocimiento didáctico-matemático de futuros profesores sobre la derivada. Estos autores demuestran que los objetos matemáticos, sus significados y los procesos identificados en las soluciones plausibles de las tareas se adaptan a los objetos matemáticos, sus significados y los procesos involucrados en las configuraciones cognitivas (análisis ontosemiótico cognitivo) asociadas a las respuestas de los profesores de formación inicial. 


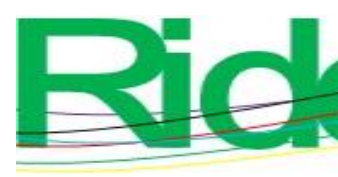

Revista Iberoamericana para la
Investigación y el Desarrollo Educativo
ISSN $2007-7467$

Asimismo, Ndjatchi (2019) diseña un instrumento para determinar los conocimientos matemáticos previos y las debilidades de un alumno de la carrera de sistemas computacionales antes de tomar el curso de números complejos. De igual manera, Morantes Moncada, Dugarte Peña y Herrera Díaz (2019) — a partir de la revisión de las habilidades de planificación, control y revisión de las actividades de aprendizaje-caracterizan el perfil de un estudiante estratégico para el análisis del cálculo diferencial en el tema funciones y sus gráficas.

Sin embargo, en numerosas investigaciones (Artigue, 1988; Rojas Maldonado, 2018, 2019, 2020b; Tall, 1995) se indica escasa capacidad para establecer una simbiosis entre la representatividad geométrica y simbólica por parte de los alumnos, habilidad esencial para la abstracción de la matemática (Ndjatchi, 2019). Peña, Murillo, Rodríguez, Cedillo y Green Arrechavala (2014) lo explican de este modo:

Los errores de los estudiantes no son casuales, se basan en conocimientos y experiencias previas, y son motivados por diferentes causas didácticas, epistemológicas, cognitivas o actitudinales. Observamos de esta forma que los errores aparecen en el trabajo de los alumnos principalmente cuando se enfrentan a conocimientos novedosos que los obligan a hacer una revisión o reestructuración de lo que ya saben (p. 48).

Durante la enseñanza y el aprendizaje, como lo menciona Ndjatchi (2019), "pueden surgir dificultades relacionadas a su propia naturaleza, otras por las circunstancias de enseñanza, otra de los profesores y su metodología didáctica” (p. 309), lo cual es congruente con lo señalado por autores como Artigue (1988), Cottrill et al. (1996), Rojas Maldonado (2018, 2019) y Tall (1995). Por ello, antes de elegir una metodología didáctica para desarrollar el quehacer educativo se debe conocer en detalle las dificultades que los alumnos enfrentan.

Resulta apropiado tener en cuenta los conocimientos iniciales de los estudiantes y establecer una relación coherente entre lo que los alumnos saben y los nuevos conocimientos. En caso contrario, se generarían dificultades innecesarias y falta de confianza de los alumnos para afrontar el nuevo conocimiento (Urbina, 2009). 


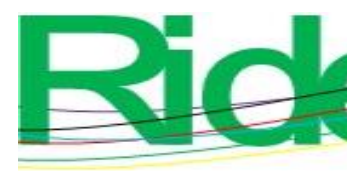

Revista Iberoamericana para la
Investigación y el Desarrollo Educativo
ISSN $2007-7467$

$\mathrm{Al}$ respecto, se debe tener en cuenta que el aprendizaje significativo surge cuando el anclaje relacional entre un contenido existente y uno nuevo se halla claro y disponible en la estructura cognitiva del estudiante (Ausubel, 1983), por lo que puede establecer similitudes y diferencias de forma no arbitraria. Por ello, Ausubel (1983) precisa lo siguiente:

La adquisición de información nueva depende en alto grado de las ideas pertinentes que ya existen en la estructura cognitiva y el aprendizaje significativo de los seres humanos ocurre a través de una interacción de la nueva información con las ideas pertinentes que ya existen en la estructura cognitiva (p. 7).

Estos conocimientos previos, como explica López Recacha (2009), son construidos de manera personal por cada individuo mientras interactúa con personas u objetos, y deben ser aprovechados para evitar el aprendizaje memorístico, el cual dista mucho del aprendizaje significativo de Ausubel. Es cierto que en muchos casos los conocimientos previos pueden ser olvidados de manera temporal, pero con un rápido recordatorio los alumnos son capaces de recobrar el significado y las implicaciones.

Ahora bien, en cuanto a los instrumentos usados para determinar los conocimientos previos de los estudiantes, primero se debe tener clara la conceptualización del constructo que se quiere evaluar, así como sus estándares de ejecución y sus respectivos indicadores de evaluación (García, 2018). Como lo comenta Soriano Rodríguez (2015), todo proceso de medición en una investigación debe trascender de la confiabilidad a la validez. Para ello, el instrumento deberá ser producto de una articulación entre paradigma, epistemología, perspectiva teórica, metodología y técnicas para la recolección y análisis de datos. En tal sentido, un instrumento es válido si mide lo que dice medir, mientras que la confiabilidad se enfoca en la probabilidad de obtener los mismos resultados.

Finalmente, un aspecto que procuran quienes realizan una investigación es la necesidad de que el instrumento seleccionado sea óptimo para recabar los datos y tenga un alto nivel de fiabilidad, en especial cuando estos basan su validez en la información verbal de percepciones, sentimientos, actitudes o conductas ( Tuapanta Dacto, Duque Vaca, A. y Mena Reinoso, 2017), de ahí que Jiménez Alfaro y Montero Rojas (2013) consideren que su diseño debe apoyarse en un proceso de perfección continua. 


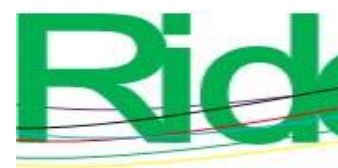

Revista Iberoamericana para la
Investigación y el Desarrollo Educativo
ISSN $2007-7467$

\section{Objetivo}

Diseñar un instrumento para determinar, en el marco de la actual emergencia sanitaria generada por el virus SARS-CoV-2, los conocimientos matemáticos previos en torno al concepto límite de alumnos del bachillerato de Ingeniería y Arquitectura (sección 1) del Colegio Primitivo y Nacional de San Nicolás de Hidalgo.

\section{Metodología}

Para el diseño del instrumento se siguieron las cuatro fases de construcción que establece Soriano Rodríguez (2015), es decir, 1) objetivos, teoría y constructo, 2) validación juicio de expertos, 3) prueba piloto y 4) validación psicométrica, las cuales se detallan a continuación:

\section{Desarrollo}

\section{Fase 1. Objetivos, teoría y constructo}

Como parte del proceso del diseño, se efectuó el análisis del programa académico de la materia Cálculo Diferencial con el fin de identificar las necesidades académicas mínimas de la unidad temática límite. Posteriormente, se creó un banco de problemas que han sido estudiados en diversas investigaciones enfocadas a la didáctica del cálculo y que han sido considerados en exámenes de admisión a nivel profesional. Luego, se seleccionaron los reactivos que satisficieran los criterios de la faceta epistémica: 1) conocimiento común 2) conocimiento especializado y 3 ) conocimiento ampliado (Loewenberg Ball, Hoover Thames y Geoffrey, 2008; Loewenberg Ball, Theule Lubienski y Spangler Mewborn, 2001).

El instrumento fue estructurado para reflejar los saberes académicos previos, como la habilidad aritmética y la habilidad algebraica, con el fin de identificar la resolución de problemas y la construcción de conocimiento matemático.

El diseño del instrumento estuvo enfocado en considerar distintos niveles de algebrización y aritmetización (Godino et al., 2015), pues se buscó la conciencia de la progresión del aprendizaje, así como alertar al docente de las dificultades pertinentes (conceptuales, proposicionales, procedimentales y argumentativas). Para ello, se procedió del siguiente modo (Godino et al., 2015): 


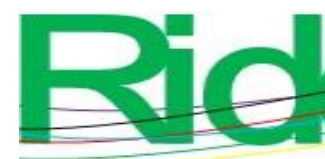

Revista Iberoamericana para la Investigación y el Desarrollo Educativo

ISSN $2007-7467$

Clasificamos el contenido que se ha de evaluar según dos variables: contenido algebraico y contenido didáctico. Para la variable contenido algebraico consideramos tres valores o categorías, en las cuales a su vez se pueden distinguir diversas subcategorías:

- Estructuras: Relación de equivalencia; propiedades de las operaciones, ecuaciones, etc.

- Funciones: Patrones aritméticos, patrones geométricos; función lineal, afín, cuadrática, etc.

- Modelización: Problemas de contexto resueltos mediante el planteamiento de ecuaciones o relaciones funcionales.

Debido a la pandemia, el instrumento fue aplicado a través de Google Forms, herramienta que permitió que los ítems se ordenaran de forma aleatoria y fueran respondidos en un tiempo limitado. En la figura 1 se estructura el conocimiento matemático común, enfocado en la faceta epistémica, es decir, reconocimiento de procesos algebraicos y aritméticos (conceptos, procedimientos, propiedades, generalización, etc.). 

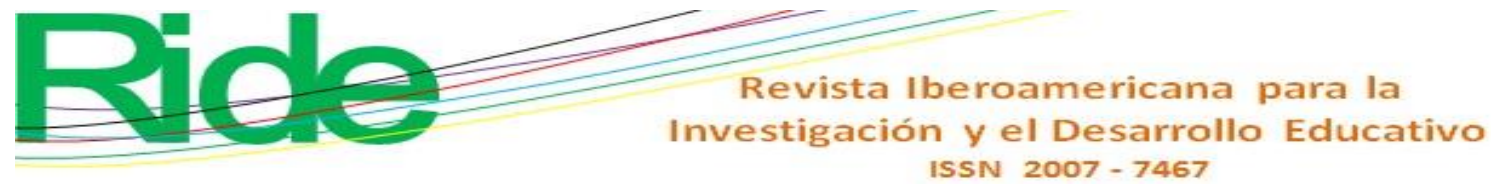

Figura 1. Conocimiento matemático común

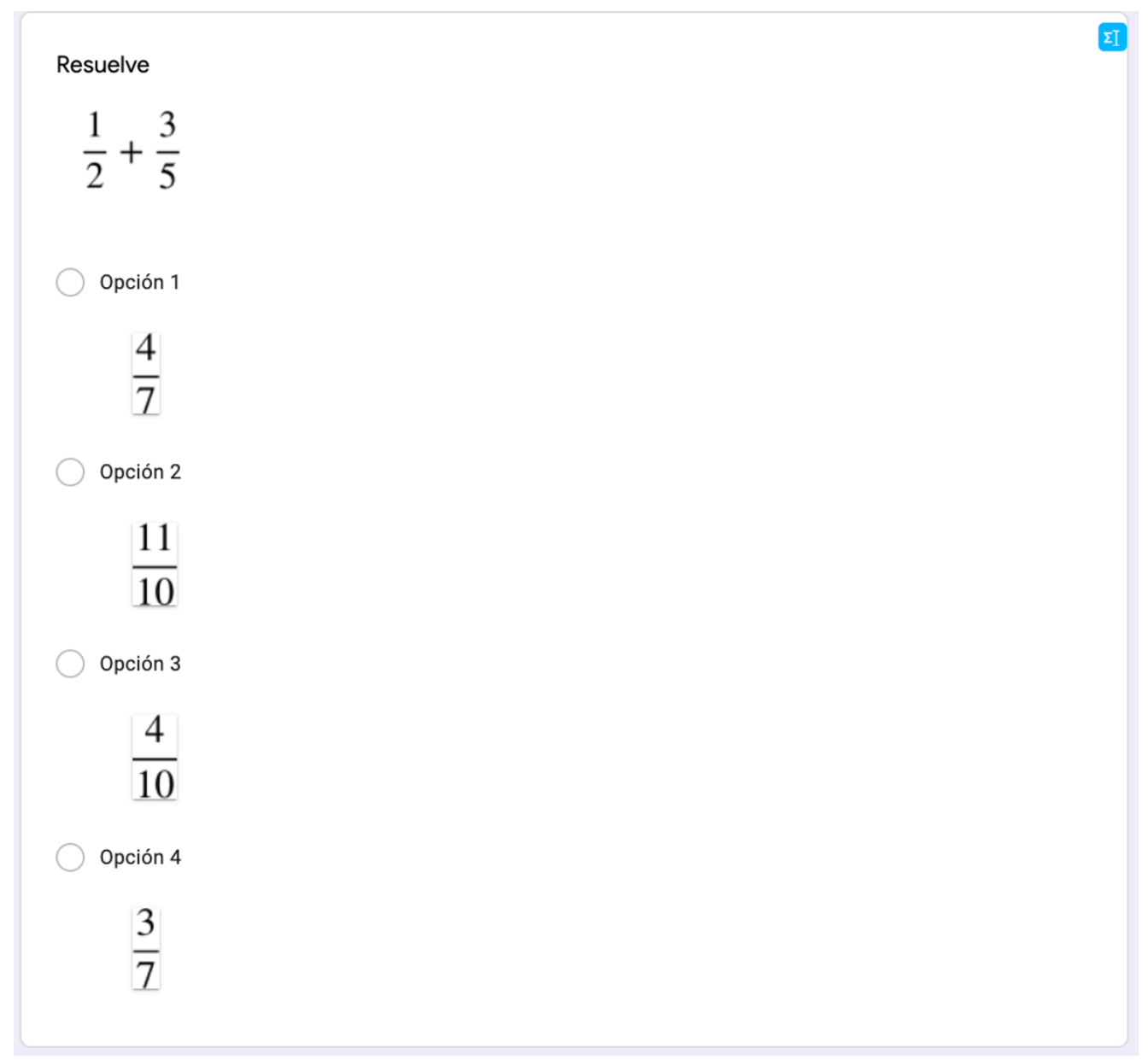

Fuente: Elaboración propia

En el anterior nivel los alumnos no requerían una capacidad de análisis o reflexión, sino seguir un mecanismo procedimental.

Por otra parte, en cuanto a las subcategorías de clasificación de contenido, estructuras y funciones de la faceta epistémica conocimiento especializado, en la figura 2 se evidencia que si bien existe un procedimiento para la resolución del problema, en realidad exige una capacidad de análisis y razonamiento, así como la identificación de distintas variables que involucran propiedades, argumentos y procedimientos.

Dicha noción favorece no solo la identificación sistemática de diferentes procedimientos de resolución, modalidades de expresión, conceptos y propiedades que se ponen en juego en su formulación, sino también la identificación de argumentaciones o justificaciones de los procedimientos y las propiedades (Pino-Fan, Godino, Castro y Font, 2012). 


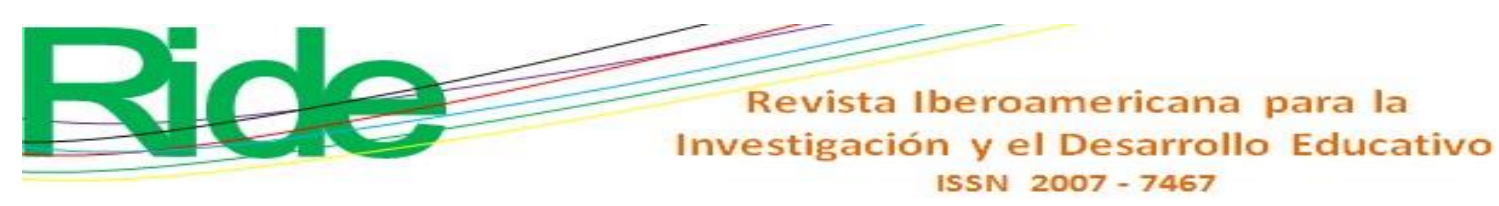

Figura 3. Proceso de composición-descomposición

Si $x$ equivale a jitomate

$$
13.6 x=(\$ 14-\$ 8.5)
$$

Fuente: Elaboración propia

Como lo indican Pino-Fan et al. (2012), "análisis similares pueden realizarse con otras resoluciones de la tarea, que pongan en juego otros procedimientos y justificaciones que involucren distintos elementos lingüísticos, conceptos y proposiciones. En este sentido los procesos de significación no tienen configuraciones únicas" (p. 431). Más adelante, en la figura 4, se muestra también claramente este proceso, el cual requiere de una mayor capacidad de síntesis, análisis y reflexión.

\section{Proceso de particularización-generalización}

En la figura 3 se partía de un hecho particular que bien puede generalizarse para cada uno de los elementos citados en la figura 2, es decir, se generaliza para cebollas, chiles, etc. Es de percatarse que está estrechamente vinculado a la transición del conocimiento común y avanzado.

\section{Proceso de materialización-idealización}

En este proceso se exploran los conocimientos específicos sobre alguna área de las matemáticas que requieren mayor capacidad de análisis y reflexión por parte del discente, como se muestra en la figura 4 , donde muchas veces se pueden contraponer ideas o conocimientos que ocasionan una disonancia. 
Figura 4. Proceso de materialización-idealización

El iPhone 11 Pro Max tiene un costo de $\$ 27,499$ pesos con IVA incluido. Si el IVA es del $16 \%$ ¿ ¿A cuánto asciende el monto correspondiente de IVA?

$\$ 4399.84$

$\$ 3,792.96$

$\$ 31,898.84$

$\$ 4231.73$

Fuente: Elaboración propia

Como lo sugiere Godino et al. (2015), en cada ítem incluimos la solución esperada, así como tres respuestas distractoras incorrectas: los objetos y los procesos que se manifiestan para la resolución, el nivel de dificultad correspondiente a la actividad académica y las categorías del conocimiento didáctico-matemático implicadas según la tabla 1.

Tabla 1. Categorías de conocimiento didáctico-matemático

\begin{tabular}{|l|c|c|c|c|c|c|}
\hline \multirow{2}{*}{\begin{tabular}{l}
\multirow{2}{*}{$\begin{array}{l}\text { Contenido } \\
\text { didáctico }\end{array}$} \\
\cline { 2 - 7 }
\end{tabular}} & \multicolumn{5}{|c|}{ Estructuras $(E)$} & \multicolumn{2}{c|}{ Funciones $(F)$} & \multicolumn{2}{c|}{ Modelización (M) } \\
\cline { 2 - 7 } & Primaria & Avanzado & Primaria & Avanzado & Primaria & Avanzado \\
\hline $\begin{array}{l}\text { Epistémico } \\
\text { (niveles de } \\
\text { algebrización) }\end{array}$ & EPI-E1 & EPI-E2 & EPI-F1 & EPI-F2 & EPI-M1 & EPI-M2 \\
\hline $\begin{array}{l}\text { Cognitivo } \\
\text { (significados } \\
\text { personales })\end{array}$ & COG-E1 & COG-E2 & COG-F1 & COG-F2 & COG-M1 & COG-M2 \\
\hline $\begin{array}{l}\text { Instruccional } \\
\text { (situaciones } y \\
\text { recursos })\end{array}$ & INS-E1 & INS-E2 & INS-F1 & INS-F2 & INS-M1 & INS-M2 \\
\hline $\begin{array}{l}\text { Contenido } \\
\text { algebraico }\end{array}$ & ALG-E1 & ALG-E2 & ALG-F1 & ALG-F2 & ALG-M1 & ALG-M2 \\
\hline
\end{tabular}

Fuente: Godino et al. (2015) 


\section{Revista Iberoamericana para la Investigación y el Desarrollo Educativo ISSN 2007 - 7467}

\section{Fase 2. Validación juicio de expertos}

Elaborados y depurados los ítems, se solicitó apoyo a tres profesores con experiencia en la materia que se encargaron de evaluar tanto la viabilidad de los reactivos como su redacción.

\section{Fase 3. Prueba piloto}

En la tabla 2 se muestra el índice de dificultad en la prueba piloto, la cual fue aplicada a 14 participantes. El índice [0.100] con 100 representa a un ítem muy fácil, donde todos los alumnos respondieron acertadamente; asimismo, se representa el error típico de los estudiantes que permite construir intervalos de confianza de las estimaciones.

Tabla 2. Índice de dificultad

\begin{tabular}{|l|l|l|l|l|l|}
\hline \multicolumn{3}{|c|}{ Momento 1: Aritmética } & \multicolumn{3}{c|}{ Momento 2: Algebra } \\
\hline Ítem & Índice de dificultad & Error típico & Ítem & Índice de dificultad & Error típico \\
\hline 1 & 79.5 & 13.6 & 1 & 68.1 & 27.7 \\
\hline 2 & 93.2 & 6.8 & 2 & 45.7 & 30.4 \\
\hline 3 & 61.9 & 14.3 & 3 & 44.7 & 23.4 \\
\hline 4 & 86 & 9.3 & 4 & 78.7 & 14.9 \\
\hline 5 & 61.4 & 36.4 & 5 & 65.2 & 19.6 \\
\hline 6 & 86.4 & 11.4 & 6 & 58.7 & 28.3 \\
\hline 7 & 97.7 & 2.3 & 7 & 41.3 & 28.3 \\
\hline 8 & 75.6 & 19.5 & 8 & 29.3 & 39 \\
\hline 9 & 47.6 & 40.5 & 9 & 67.4 & 17.4 \\
\hline 10 & 63.6 & 36.4 & & & \\
\hline 11 & 56.1 & 17.1 & & & \\
\hline 12 & 93.2 & 4.5 & & & \\
\hline 13 & 60.5 & 23.3 & & & \\
\hline 14 & 6.8 & 65.9 & & & \\
\hline
\end{tabular}

Fuente: Elaboración propia 


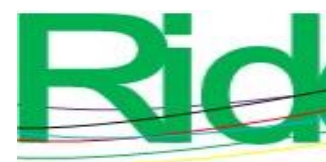

Revista Iberoamericana para la
Investigación y el Desarrollo Educativo
ISSN $2007-7467$

\section{Fase 4. Validación psicométrica}

En esta fase se aseguró el funcionamiento de cada uno de los ítems, así como su descarte (como se muestra en la tabla 2). Luego se procedió al cálculo del coeficiente de fiabilidad de consistencia interna (alfa de Cronbach) a través del software SPSS (versión 25). Como se muestra en la tabla 3, se consiguió un coeficiente de 0.807 (muy buena confiabilidad), lo que indica que estos resultados se pueden reproducir en diferentes muestras sin que constituyan un gran sesgo por parte de los participantes. Además, los puntajes de cada pregunta fueron consistentes con el puntaje total del instrumento.

Tabla 3. Estadística de fiabilidad

\begin{tabular}{|c|c|}
\hline Alfa de Cronbach & N. ${ }^{\circ}$ de elementos \\
\hline .807 & 14 \\
\hline
\end{tabular}

Fuente: Elaboración propia

En la figura 5 se observa cómo el instrumento fue sometido a la prueba de unidimensionalidad del constructo a través del estudio del gráfico de sedimentación en el programa SPSS.

Figura 5. Gráfico de sedimentación del cuestionario piloto

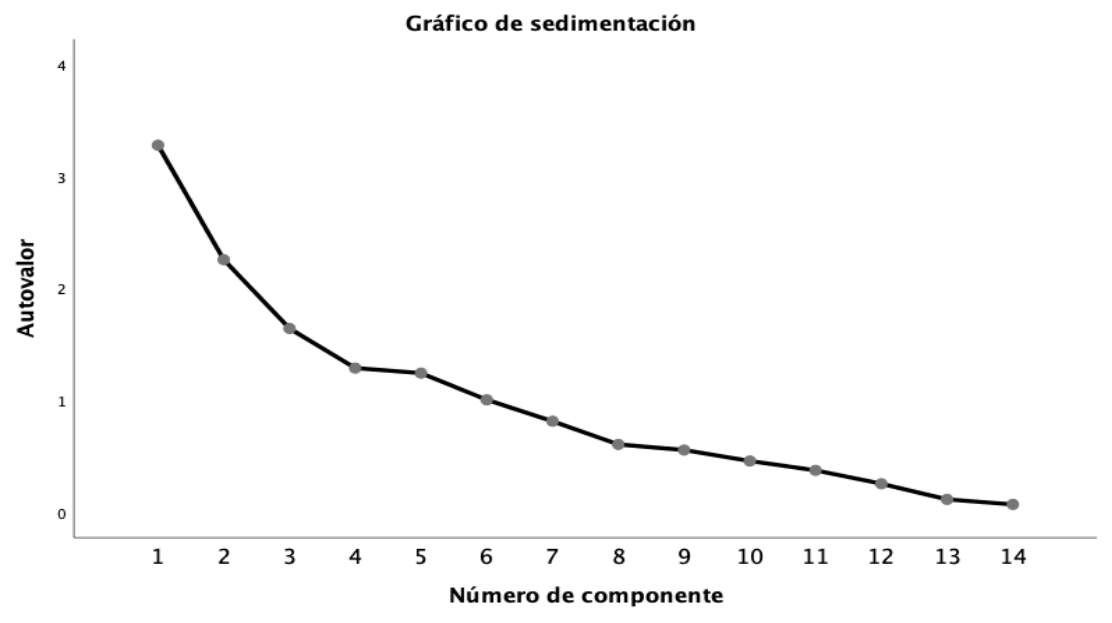

Fuente: Elaboración propia

Finalmente, en la tabla 4 se muestra el porcentaje de varianza del primer componente, que fue mayor a 20, así como el resto menores a 10 (excepto el 2 y 3), por lo que no puede ser considerado como un instrumento multidimensional. 
Tabla 4. Análisis de varianza de prueba piloto

\begin{tabular}{|c|c|c|c|c|c|c|c|c|c|}
\hline \multicolumn{10}{|c|}{ Varianza total explicada } \\
\hline & \multicolumn{3}{|c|}{ Autovalores iniciales } & \multicolumn{3}{|c|}{$\begin{array}{c}\text { Sumas de cargas al } \\
\text { cuadrado de la } \\
\text { extracción }\end{array}$} & \multicolumn{3}{|c|}{$\begin{array}{c}\text { Sumas de cargas al } \\
\text { cuadrado de la rotación }\end{array}$} \\
\hline $\begin{array}{l}\text { Compone } \\
\text { nte }\end{array}$ & $\begin{array}{l}\text { Tot } \\
\text { al }\end{array}$ & $\begin{array}{l}\% \text { de } \\
\text { varian } \\
\text { za }\end{array}$ & $\begin{array}{l}\% \\
\text { acumula } \\
\text { do }\end{array}$ & $\begin{array}{l}\text { Tot } \\
\text { al }\end{array}$ & $\begin{array}{l}\% \text { de } \\
\text { varian } \\
\text { za }\end{array}$ & $\begin{array}{l}\% \\
\text { acumula } \\
\text { do }\end{array}$ & $\begin{array}{l}\text { Tot } \\
\text { al }\end{array}$ & $\begin{array}{l}\% \text { de } \\
\text { varian } \\
\text { za }\end{array}$ & $\begin{array}{l}\% \\
\text { acumula } \\
\text { do }\end{array}$ \\
\hline 1 & $\begin{array}{l}3.27 \\
8\end{array}$ & $\begin{array}{l}23.41 \\
2\end{array}$ & 23.412 & $\begin{array}{l}3.27 \\
8\end{array}$ & $\begin{array}{l}23.41 \\
2\end{array}$ & 23.412 & $\begin{array}{l}2.45 \\
4\end{array}$ & $\begin{array}{l}17.52 \\
6\end{array}$ & 17.526 \\
\hline 2 & $\begin{array}{l}2.25 \\
7\end{array}$ & $\begin{array}{l}16.11 \\
8\end{array}$ & 39.530 & $\begin{array}{l}2.25 \\
7\end{array}$ & $\begin{array}{l}16.11 \\
8\end{array}$ & 39.530 & $\begin{array}{l}2.25 \\
7\end{array}$ & $\begin{array}{l}16.12 \\
1\end{array}$ & 33.647 \\
\hline 3 & $\begin{array}{l}1.64 \\
4\end{array}$ & $\begin{array}{l}11.74 \\
2\end{array}$ & 51.272 & $\begin{array}{l}1.64 \\
4\end{array}$ & $\begin{array}{l}11.74 \\
2\end{array}$ & 51.272 & $\begin{array}{l}1.67 \\
7\end{array}$ & $\begin{array}{l}11.98 \\
1\end{array}$ & 45.627 \\
\hline 4 & $\begin{array}{l}1.29 \\
0\end{array}$ & 9.216 & 60.488 & $\begin{array}{l}1.29 \\
0\end{array}$ & 9.216 & 60.488 & $\begin{array}{l}1.60 \\
9\end{array}$ & $\begin{array}{l}11.49 \\
5\end{array}$ & 57.122 \\
\hline 5 & $\begin{array}{l}1.24 \\
5\end{array}$ & 8.895 & 69.383 & $\begin{array}{l}1.24 \\
5\end{array}$ & 8.895 & 69.383 & $\begin{array}{l}1.37 \\
3\end{array}$ & 9.807 & 66.928 \\
\hline 6 & $\begin{array}{l}1.00 \\
7\end{array}$ & 7.195 & 76.578 & $\begin{array}{l}1.00 \\
7\end{array}$ & 7.195 & 76.578 & $\begin{array}{l}1.35 \\
1\end{array}$ & 9.650 & 76.578 \\
\hline 7 & .816 & 5.829 & 82.407 & & & & & & \\
\hline 8 & .610 & 4.356 & 86.763 & & & & & & \\
\hline 9 & .560 & 3.998 & 90.762 & & & & & & \\
\hline 10 & .462 & 3.299 & 94.061 & & & & & & \\
\hline 11 & .379 & 2.704 & 96.765 & & & & & & \\
\hline 12 & .259 & 1.848 & 98.614 & & & & & & \\
\hline 13 & .120 & .855 & 99.468 & & & & & & \\
\hline 14 & .074 & .532 & 100.000 & & & & & & \\
\hline
\end{tabular}

Fuente: Elaboración propia 


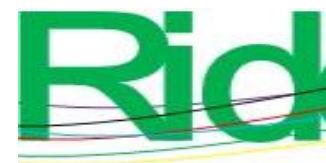
Revista Iberoamericana para la
Investigación y el Desarrollo Educativo
ISSN $2007-7467$

\section{Conclusiones}

La elaboración del instrumento analizado en este trabajo puede ser una herramienta muy útil no solo para detectar los conocimientos previos que los alumnos tienen en cuanto al cálculo diferencial, sino principalmente para crear estrategias didácticas que permitan atender esas debilidades a partir de los estilos de aprendizaje de los estudiantes. Además, se debe prever que, debido a la pandemia, estamos viviendo una época en donde las clases presenciales han sido sustituidas por encuentros virtuales, los cuales exigen métodos de enseñanza y aprendizaje diferentes a los tradicionales.

Para el alumno, por tanto, este instrumento también puede representar una oportunidad no solo para conocer sus debilidades en la materia, sino para emprender acciones que permitan cambiar esa realidad. Al respecto, los estudiantes deben ser conscientes de la responsabilidad que tienen en sus procesos de aprendizaje, lo cual se ha convertido en una necesidad para encarar los retos que han surgido en estos tiempos de covid-19. En efecto, la pandemia evidenció que no basta solo con aprobar las asignaturas, pues el aprendizaje es una labor diaria que demanda mucho esfuerzo independiente, así como, lógicamente, la aplicación de estrategias idóneas que se ajusten a esta nueva realidad.

\section{Futuras líneas de investigación}

En primer lugar, se considera prioritaria la creación de diversos instrumentos que permitan al docente diagnosticar el nivel de conocimientos que sus alumnos tienen en determinada materia, pues de ese modo se podrán implementar estrategias que atiendan esas necesidades (Rojas Maldonado, 2015). Sin embargo, después de desarrollar esas actividades propuestas también será indispensable que se efectúen investigaciones para determinar si con ellas se consiguieron los resultados esperados. 


\section{Referencias}

Artigue, M. (1988). Ingénierie didactique. Recherches en Didactique des Mathematiques, 9(3), 281-308.

Ausubel, D. (1983). Teoría del aprendizaje significativo. En Fascículos de CEIF (vol. 1) (pp. 1-10). Doi: https://doi.org/10.1007/978-3-540-74459-7_8

Consejo Nacional de Evaluación [Coneval] (2018). Pobreza en México. Recuperado de https://www.coneval.org.mx/Medicion/MP/Paginas/Pobreza-2018.aspx

Cornu, B. (1991). Limits. In Tall, D. (ed.), Advanced Mathematical Thinking (pp. 153-166). Dordrecht: Kluwer.

Cottrill, J., Dubinsky, E., Nichols, D., Schwingendorf, K., Thomas, K. and Vidakovic, D. (1996). Understanding the limit concept: Beginning with a coordinated process scheme. Journal of Mathematical Behavior. Doi: https://doi.org/10.1016/S07323123(96)90015-2

García, R. (2018). Diseño y construcción de un instrumento de evaluación de la competencia matemática: aplicabilidad práctica de un juicio de expertos. Ensaio: Avaliação e Políticas Públicas em Educação, 26(99), 347-372. Recuperado de http://www.scielo.br/scielo.php?script=sci_arttext\&pid=S0104$40362018000200347 \& \operatorname{lng}=\mathrm{es} \& \operatorname{lng}=\mathrm{es}$

Godino, J. D., Aké, L. P., Contreras, Á., Díaz, C., Estepa, A., Blanco, T. F., ... Wilhelmi, M. R. (2015). Designing a questionnaire for assessing the didactic-mathematical knowledge on elementary algebraic reasoning. Ensenanza de las Ciencias, 33(1), 127150. Doi: https://doi.org/10.5565/rev/ensciencias. 1468

Hodges, C., Moore, S., Lockee, B., Trust, T. and Bond, A. (2020). La diferencia entre la enseñanza remota de emergencia y el aprendizaje en línea. En Enseñanza remota de emergencia: textos para la discucisón (pp. 10-22). The Learning Factor.

Instituto Nacional de Estadística Geografía e Informática [Inegi] (2018). Encuesta Nacional sobre la Disponibilidad y Uso de Tecnologías de la Información en los Hogares. Recuperado de https://www.inegi.org.mx/programas/dutih/2018/

Jiménez Alfaro, K. y Montero Rojas, E. (2013). Aplicación del modelo de Rasch, en el análisis psicométrico de una prueba de diagnóstico en matemática. Revista Digital: Matemática, Educación $\quad$ e Internet, $13(1), \quad$ 1-24. Doi: 


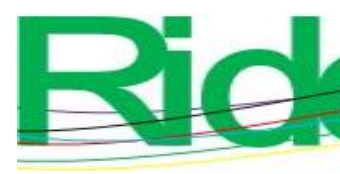

Revista Iberoamericana para la
Investigación y el Desarrollo Educativo
ISSN $2007-7467$

https://doi.org/10.18845/rdmei.v13i1.1628

Loewenberg Ball, D., Hoover Thames, M. and Geoffrey, P. (2008). Content Knowledge for Teaching. What Makes It Special? Journal of Teacher Education, 59(5), 389-407. Doi: https://doi.org/10.1016/B978-0-08-044894-7.00642-4

Loewenberg Ball, D., Theule Lubienski, S. and Spangler Mewborn, D. (2001). Research on teaching mathematics: The unsolved problem of teachers' mathematicak knowledge. Handbook of Research on Teaching, 433-456. Retrieved from http://wwwpersonal.umich.edu/ dball/chapters/BallLubienskiMewbornChapter.pdf

López Recacha, J. A. (2009). La importancia de los conocimientos previos para el aprendizaje de nuevos contenidos. Innovación y Experiencias Educativas, 1-14. Recuperado de http://www.csicsif.es/andalucia/modules/mod_ense/revista/pdf/Numero_16/JOSE ANTONIO_LOPEZ_1.pdf

Morantes Moncada, G., Dugarte Peña, E. y Herrera Díaz, J. (2019). Perfil del aprendiz estratégico para el estudio de cálculo diferencial mediado por las TIC. Revista Logos, Ciencia \& Tecnología, 11(3), 152-167. Doi: https://doi.org/10.22335/rlct.v11i3.864

Mota Villegas, D. y Valles Pereira, R. (2015). Papel de los conocimientos previos en el aprendizaje de la matemática universitaria. Acta Scientiarum. Education, 37(1). Doi: https://doi.org/10.4025/actascieduc.v37i1.21040

Ndjatchi, M. K. C. (2019). Conocimientos previos de números complejos en ingeniería. Ciencia, Docencia y Tecnología, 30(58), 305-329. Doi: https://doi.org/10.33255/3058/477

Organización de las Naciones Unidas para la Educación, la Ciencia y la Cultura [Unesco] (2020). Impacto de la covid-19 en la educación. Recuperado de https://es.unesco.org/covid19/educationresponse

Peña, A., Murillo, D., Rodríguez, C., Cedillo, N. y Green Arrechavala, I. (2014). Limitaciones que presentan los estudiantes de noveno grado del año 2013, del CIIE en la resolución de problema aplicados relacionado con ecuaciones lineales. Revista Académica Paradigma Estudiantil, 1(1), 46-50.

Pino-Fan, L. R., Godino, J. D. y Vincenç, F. (2013). Diseño y aplicación de un instrumento para explorar la faceta epistémica del conocimiento didáctico-matemático de futuros 


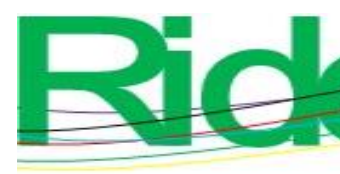

Revista Iberoamericana para la Investigación y el Desarrollo Educativo ISSN $2007-7467$

profesores sobre la derivada (segunda parte). Revemat, 8, 1-47.

Pino-Fan, L. R., Godino, J. D., Castro, W. F. and Font, V. (2012). Conocimiento didácticomatemático de profesores en formación: explorando el conocimiento especializado sobre la derivada. En SEIEM (ed.), Investigación en educación matemática XVI (pp. 427-434). Jaén.

Rojas Maldonado, E. R. (2015). Secuencias didácticas para la enseñanza del concepto de límite en el cálculo. Revista Internacional de Aprendizaje en Ciencia, Matemáticas y Tecnoloǵla, 2(2), 63-76. Recuperado de http://funes.uniandes.edu.co/15392/1/Rojas2016Secuencias.pdf

Rojas Maldonado, E. R. (2016). Resultados de la aplicación de secuencias didácticas para la comprensión del concepto del límite en el bachillerato Nicolaíta. RIDE Revista Iberoamericana para la Investigación y el Desarrollo Educativo. Doi: https://doi.org/10.23913/ride.v6i12.227

Rojas Maldonado, E. R. (2018). Mathematization: A teaching strategy to improve the learning of Calculus. Revista Iberoamericana para la Investigación y el Desarrollo Educativo, 9(17).

Rojas Maldonado, E. R. (2019). Diseño de estrategia de apertura para la interpretación gráfica-analítica a través de Desmos como preparación para el aprendizaje del cálculo diferencial. RIDE Revista Iberoamericana para la Investigación y el Desarrollo Educativo, 10(19). Doi: https://doi.org/10.23913/ride.v10i19.493

Rojas Maldonado, E. R. (2020a). Análisis de la percepción de los profesores en activo referente al uso de la tecnología en la Matemática. Polyphōnia. Revista de Educación Inclusiva, 4(2), 167-196. Recuperado de http://revista.celei.cl/index.php/PREI/index

Rojas Maldonado, E. R. (2020b). Understanding Fundamental Concepts of Calculus Through Desmos. An Intervention. RIDE Revista Iberoamericana para la Investigación y el Desarrollo Educativo, 10(20), 1-15. https://doi.org/https://doi.org/10.23913/ride.v10i20.672

Soriano Rodríguez, A. M. (2015). Diseño y validación de instrumentos de medición. Diálogos, (14). Doi: https://doi.org/10.5377/dialogos.v0i14.2202

Tall, D. (1992). The transition to advanced mathematical thinking: Functions, limits, infinity and proof. In Grouws, D. A. (ed.), Handbook of Research on Mathematics Teaching 
and Learning. Macmillan, New York, 495-511. Retrieved from http://scholar.google.com/scholar?hl=en\&btnG=Search\&q=intitle:The+Transition+to+ Advanced+Mathematical+Thinking+:+Functions,+Limits,+Infinity+and+Proof\#0

Tall, D. (1995). Cognitive growth in elementary and advanced mathematical thinking. PME conference, 1(July), 1-61.

Tuapanta Dacto, J. V., Duque Vaca, A. y Mena Reinoso, A. P. (2017). Alfa de Cronbach para validar un cuestionario de uso de TIC en docentes universitarios. mktDescubreESPOCH FADE, (10), 37-48.

Urbina, M. (2009). Los conocimientos previos y su importancia para la comprensión del lenguaje matemático en la educación superior. Universidad, Ciencia y Tecnología, 13(52), 1-12. Recuperado de http://ve.scielo.org/scielo.php?script=sci_arttext\&pid=S1316-48212009000300004 


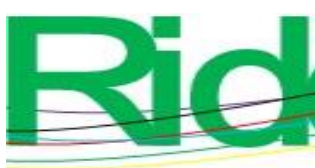

Revista Iberoamericana para la Investigación y el Desarrollo Educativo

ISSN 2007 - 7467

\begin{tabular}{|l|l|}
\hline Rol de Contribución & Autor (es) \\
\hline Conceptualización & Erick Radaí Rojas Maldonado \\
\hline Metodología & Erick Radaí Rojas Maldonado \\
\hline Software & Erick Radaí Rojas Maldonado \\
\hline Validación & Jacqueline Toscano \\
\hline Análisis Formal & Erick Radaí Rojas Maldonado \\
\hline Investigación & Erick Radaí Rojas Maldonado y Jacqueline Toscano Galenana \\
\hline Recursos & Jacqueline Toscano Galenana \\
\hline Curación de datos & Jacqueline Toscano Galenana \\
\hline $\begin{array}{l}\text { Escritura - Preparación del } \\
\text { borrador original }\end{array}$ & Jacqueline Toscano Galenana \\
\hline $\begin{array}{l}\text { Escritura - Revisión y } \\
\text { edición }\end{array}$ & Erick Radaí Rojas Maldonado \\
\hline Visualización & Erick Radaí Rojas Maldonado \\
\hline Supervisión & Erick Radaí Rojas Maldonado \\
\hline Administración de Proyectos & Erick Radaí Rojas Maldonado \\
\hline Adquisición de fondos & Erick Radaí Rojas Maldonado \\
\hline
\end{tabular}

